\title{
Role of oral dehydroepiandrosterone in diminished ovarian function
}

\author{
Meena Dayal, Preeti Yadav*, Amrita Chaurasia, Urvashi Singh, Veena Gupta, Shweta Patel
}

Department of obstetrics and gynecology, Moti Lal Nehru Medical College, Allahabad, UP, India

Received: 19 January 2016

Revised: 27 January 2016

Accepted: 16 February 2016

\author{
*Correspondence: \\ Dr. Preeti Yadav, \\ E-mail: preeti_yadav3@yahoo.com
}

Copyright: () the author(s), publisher and licensee Medip Academy. This is an open-access article distributed under the terms of the Creative Commons Attribution Non-Commercial License, which permits unrestricted non-commercial use, distribution, and reproduction in any medium, provided the original work is properly cited.

\begin{abstract}
Background: Ovarian reserve decreases with age and this study determine the effect of oral DHEA supplementation on S.AMH concentration and conception rate in women with diminished ovarian function.

Methods: The study was conducted on 72 infertile women of $20-45$ years age who had S.AMH $<2.2 \mathrm{ng} / \mathrm{ml}$, which was taken as cut off for diminished ovarian reserve in the study. The cases of study group received oral DHEA $25 \mathrm{mg}$ TDS and cases of control group received placebo (folic acid $5 \mathrm{mg}$ OD) for 12 weeks. Day 2 S.AMH, S.FSH, S.LH, S.E2, S. Inhibin B, ovarian volume and AFC were measured at first visit and after 12 weeks of oral DHEA or placebo. Results: After 12 weeks of oral DHEA supplementation, S. AMH concentration was found to be significantly improved $(p=0.01)$. S.FSH was decreased $(p=0.04)$ and $S . E_{2}$ was increased $(p=0.03)$. S. LH showed insignificant decrease. S. Inhibin B, ovarian volume and AFC were increased but insignificantly. Improvement in ovarian reserve resulted in significantly higher pregnancy rate $(p=0.04)$ and live births $(p=0.04)$ in women treated with DHEA than in the control group. Miscarriage rate was found to be decreased but insignificantly.

Conclusions: DHEA improves the ovarian reserve.
\end{abstract}

Keywords: Serum antimullerian hormone, Serum follicular stimulating hormone, Serum luteinizing hormone, Serum estradiol, Antral follicular count, Dehydroepiandrosterone

\section{INTRODUCTION}

Ovarian factors are responsible for $20-40$ per cent cases of infertility. Ageing is associated with a decline in the number of ovarian follicles, menstrual irregularities, ovarian hormonal deficiency, anovulation, decreased fertility and finally, menopause, usually occurring at a mean age of 51 years. Ovarian reserve describes a woman's reproductive potential with respect to ovarian follicle number and oocyte quality. Total ovarian reserve decreases with time due to continuous follicle recruitment. ${ }^{1}$ Diminished ovarian reserves (DOR) is defined as the follicle pool, at any given age, smaller than expected. Older ovaries have few antral follicles and high rates of atresia. Androgens play a major role in follicular maturation. There is high concentration of androgen receptors in preantral and antral stages. They stimulate granulosa cells and are important for ovarian steroidogenesis. $^{2,3}$ DHEA can improve steroidogenesis since it is a precursor of estrogen and testosterone. It may also influence ovarian follicular growth by increase in IGF-1, that in turn stimulating mitosis, proliferation of the granulosa cells and production of AMH as well. This optimizes the hormonal feedback to the pituitary gland leading to adequate response of the pituitary FSH and thus inducing normal oocyte maturation and good oocyte quality. The latest evidences suggest a new concept, ovarian environment and the synergism between androgens and FSH in early stages of follicular maturation. DHEA progressively improves ovarian reserve by improving ovarian environment as nicely as lowers miscarriage chances in DOR individuals. ${ }^{4,5}$ This study was conducted with aim to determine the effect of oral DHEA supplementation on ovarian reserve and conception rate in women with DOR. 


\section{METHODS}

The present study was carried out on 72 infertile women of 20-45 years of age, over a period of twelve months. This study was a prospective study and cases were divided into study group and control group. Women in reproductive age group with more than 1 year of infertility with S.AMH $<2.2 \mathrm{ng} / \mathrm{ml}$, which was taken as cut off for diminished ovarian reserve, who had regular menstruation, normal hysterosalpingogram (HSG) and normal semen analysis of husband were included in study. The cases with abnormal HSG, baseline FSH/E within normal age specific criteria, polycystic ovarian syndrome, medical conditions that would contraindicate pregnancy or ovulation induction or general anesthesia and desire to undergo other fertility treatments before completing three months of study were excluded. A detailed history was taken with special reference to age, parity, socio-economic status, education, occupation and personal habits. The husband and wife were equally questioned separately and then together, with special reference to duration of marriage, previous marriage, coital difficulty, separation for significant period of time and type of contraception used. Detailed history about any previous illness, appendicitis, peritonitis, tuberculosis in any site or operation on or near the genital tract, abdominal or CNS operations was taken for all cases. Careful past history of hypertension, diabetes and other endocrine disease or any other chronic illness was taken. Details of menstrual history regarding menarche, regularity of menstruation, oligomenorrhoea, menorrhagia and dysmenorrhoea was asked. History of drug intake like mefenamic acid, which may interfere with ovulation and about alcohol intake, was taken. History of galactorrhoea, hirsutism and weight gain was taken. A detailed history from husband was taken to rule out male factors that are responsible for infertility with special reference to personal habits, occupation and any previous illness. Family history of infertility, premature ovarian failure, congenital and developmental defects, mental retardation and chronic illness was asked. A thorough general, systemic and gynaecological examination was done.

Apart from routine investigations of infertility, estimation of S. FSH, S. LH, S. Estradiol $\left(\mathrm{E}_{2}\right), \mathrm{S}$. AMH and $\mathrm{S}$. Inhibin B was done on day 2 of menstrual cycle. S. FSH, $\mathrm{S}$. LH and $\mathrm{S}$. $\mathrm{E}_{2}$ levels were measured by solid phase, two site, chemluminescent immunometric assay. Inhibin B was tested by ELISA method. AMH was estimated by sandwich enzyme immunoassay method. Baseline ultrasonography on day 2 was done to identify pretreatment antral follicular count (AFC) and ovarian volume by using $7.5 \mathrm{MHz}$ transvaginal probe. Examination of ovary was established by scanning from the outer to inner margin. All follicles of $2-10 \mathrm{~mm}$ size were measured and counted in each ovary. The sum of both counts was demonstrated as AFC. The volume of left and right ovary was assessed by measuring the diameter of the contour in three perpendicular directions and applying the equation of volume of an ellipsoid to calculate ovarian volume $\left(\mathrm{D}_{1} \mathrm{xD}_{2} \times \mathrm{D}_{3} \times 0.523\right)$. Total ovarian volume was then obtained by sum of the volume of left and right ovary.

The cases that had $\mathrm{S} . \mathrm{AMH}<2.2 \mathrm{ng} / \mathrm{ml}$ were selected for the study. Out of 72 cases, 36 cases in study group were given oral DHEA supplementation $25 \mathrm{mg}$ thrice a day and 36 cases in control group were given placebo (folic acid, $5 \mathrm{mg}$ once a day) treatment for a period of 12 weeks. After 12 weeks of DHEA supplementation, the effect was observed for any improvement in day 2 serum FSH, LH, E $E_{2}, \mathrm{AMH}$ and inhibin B levels. The AFC and ovarian volume was also observed for any improvement after 12 weeks of oral DHEA administration. The cases either conceived spontaneously or after ovulation induction after 12 weeks of DHEA/placebo supplementation and were followed up for one year. Both the groups were followed up to observe for pregnancy, miscarriage and live birth. The research protocol was approved by institutional ethics committee before the study began and all human participants gave informed consent before the study began.

For all statistical analysis $\mathrm{p}<0.05$ was considered as significant. The chi-square test was carried out for categorical variables and descriptive statistics were given as the mean $\pm \mathrm{SD}$.

\section{RESULTS}

The cases were divided into three groups according to age. Majority cases of study group $(53.84 \%)$ and control group (50.00\%) belonged to 21-30 years of age (Table 1). The difference between study and control groups regarding habitat, socioeconomic status, educational status, occupation, age at marriage and duration of infertility was found to be statistically insignificant. After 12 weeks of oral DHEA supplementation, S. FSH levels were decreased from $12.13 \pm 2.67 \mathrm{mIU} / \mathrm{ml}$ to $3.56 \pm 1.2$ $\mathrm{mIU} / \mathrm{ml}$ and this decrease was statistically significant $(\mathrm{p}=0.04)$. In DHEA treated group, S. LH levels were minimally decreased from $9.37 \pm 3.02 \mathrm{mIU} / \mathrm{ml}$ to $8.83 \pm$ $2.75 \mathrm{mIU} / \mathrm{ml}$ but was statistically insignificant. The $\mathrm{S} . \mathrm{E}_{2}$ levels showed significant increase from $29.66 \pm 4.9 \mathrm{pg} / \mathrm{ml}$ to $57.67 \pm 9.6 \mathrm{pg} / \mathrm{ml}(\mathrm{p}=0.03)$. S. AMH levels showed significant increase from $0.65 \pm 0.30 \mathrm{ng} / \mathrm{ml}$ to $1.43 \pm 0.57$ $\mathrm{ng} / \mathrm{ml}(\mathrm{p}=0.01)$ (Table 2). S. Inhibin B levels showed minimal increase from $34.06 \pm 3.61 \mathrm{pg} / \mathrm{ml}$ to $37.67 \pm 4.51$ $\mathrm{pg} / \mathrm{ml}$ but this increase was statistically insignificant. Increase in AFC although statistically insignificant was observed in DHEA treated group from 6.95 1.61 to $13.05 \pm 1.73$ (Table 3). No significant change was observed in ovarian volume in DHEA treated group. The cases either conceived spontaneously or after ovulation induction after 12 weeks of DHEA/placebo supplementation and were followed up for one year. Pregnancy rate was found significantly higher $49.66 \%$ in DHEA treated group as compared to $13.66 \%$ in placebo group $(\mathrm{p}=0.04)$ (Table 4). Decrease in miscarriage rate 
was found, it was $16.78 \%$ in placebo group and $9.13 \%$ in DHEA treated group. The live birth rate in DHEA treated group was $31.74 \%$ which was significantly more than the live birth rate found in placebo group $2.56 \%(\mathrm{p}=0.04)$.

Table 1: Age distribution.

\begin{tabular}{|lllll|}
\hline \multirow{2}{*}{ Age Group (in years) } & \multicolumn{2}{l|}{ Study Group $\{\mathbf{N}=36\}$} & \multicolumn{2}{l|}{ Control Group $\{\mathbf{N}=36\}$} \\
\hline $21-30$ (Group 1) & NO. & $\%$ & NO. & $\%$ \\
\hline $31-40$ (Group 2) & 19 & 53.84 & 18 & 50.00 \\
\hline$>40$ (Group 3) & 13 & 34.61 & 15 & 42.30 \\
\hline Total & 4 & 11.53 & 3 & 7.69 \\
\hline MEAN \pm S.D. & 36 & 100 & 36 & 100 \\
\hline
\end{tabular}

Table 2: Effect of DHEA on hormone levels.

\begin{tabular}{|llllll|} 
& Study group & & Control group & \multicolumn{2}{c|}{ p Value } \\
& Pre Tt & Post Tt & Pre Tt & Post Tt & 0.01 \\
\hline S. AMH $(\mathrm{ng} / \mathrm{ml})$ & $0.65 \pm 0.30$ & $1.43 \pm 0.57$ & $0.76 \pm 0.37$ & $0.83 \pm 0.42$ & 0.04 \\
\hline S. FSH $(\mathrm{mIU} / \mathrm{ml})$ & $12.13 \pm 2.67$ & $3.56 \pm 1.2$ & $11.37 \pm 2.45$ & $10.56 \pm 2.25$ & 0.86 \\
\hline S. LH $(\mathrm{mIU} / \mathrm{ml})$ & $9.37 \pm 3.02$ & $8.83 \pm 2.75$ & $10.1 \pm 4.5$ & $10.61 \pm 2.99$ & 0.03 \\
\hline S. E2 $(\mathrm{pg} / \mathrm{ml})$ & $29.66 \pm 4.9$ & $57.67 \pm 9.6$ & $28.66 \pm 4.5$ & $30.01 \pm 4.3$ & 1 \\
\hline S. Inhibin B $(\mathrm{pg} / \mathrm{ml})$ & $34.06 \pm 3.61$ & $37.67 \pm 4.51$ & $36.11 \pm 5.57$ & $37.87 \pm 5.10$ & 1 \\
\hline
\end{tabular}

Table 3: Effect of DHEA on AFC and ovarian volume.

\begin{tabular}{|llllll|}
\hline & Study group & & \multicolumn{2}{c|}{ Control group } & \multicolumn{2}{c|}{ p Value } \\
\cline { 2 - 4 } & Pre Tt & Post Tt & Pre Tt & Post Tt & \\
\hline AFC & $6.95 \pm 1.61$ & $13.05 \pm 1.73$ & $7.59 \pm 1.02$ & $7.84 \pm 0.90$ & 0.56 \\
\hline Ovarian volume(ml) & $6.30 \pm 0.95$ & $7.81 \pm 0.98$ & $6.63 \pm 0.45$ & $6.70 \pm 0.75$ & 0.9 \\
\hline
\end{tabular}

Table 4: Effect of DHEA on Pregnancy Outcome.

\begin{tabular}{|c|c|c|c|c|c|c|c|}
\hline \multirow{2}{*}{ Variable } & \multicolumn{2}{|c|}{ Group 1 (21-30 years) } & \multicolumn{2}{|c|}{ Group 2 (31-40 years) } & \multicolumn{2}{|c|}{ Group 3 (>40 years) } & \multirow[t]{2}{*}{ p value } \\
\hline & Study & Control & Study & Control & Study & Control & \\
\hline Pregnancy rate & $64.28 \%$ & $23.07 \%$ & $66.66 \%$ & $18.18 \%$ & $33.33 \%$ & - & 0.04 \\
\hline Miscarriage rate & $7.14 \%$ & $7.69 \%$ & - & $9.01 \%$ & - & - & 0.44 \\
\hline Live birth rate & $28.57 \%$ & $7.69 \%$ & $33.33 \%$ & - & $33.33 \%$ & - & 0.04 \\
\hline
\end{tabular}

\section{DISCUSSION}

Ovarian ageing, as manifested by decreased ovarian reserve, is responsible for age related decline in fertility and of increase in adverse reproductive events such as miscarriages and aneuploid pregnancies. During the past two decades, a greater majority of women have been known to plan their pregnancy in their late twenties and early thirties; as a result they may experience consequences of declining fecundity. The decrease in fertility with ageing is due to decreasing number of primordial follicles after birth. A good quality egg releases certain substances (e.g. inhibin-B, estrogen) that suppress the FSH level (negative feedback).When the egg quality is compromised, these negative feedback signals are weak leading to increase in FSH levels. With ovarian ageing, the first change is a decrease in AMH levels, followed by a decline in inhibin-B and finally by an increase in FSH levels.

In present study, the mean S. FSH levels decreased from $25.03 \pm 8.46 \mathrm{mIU} / \mathrm{ml}$ to $8.5 \pm 3.3 \mathrm{mIU} / \mathrm{ml}$ after 12 weeks of treatment with oral DHEA and was statistically significant $(\mathrm{p}=0.04)$. In accordance with present study, Sonmezer et al also assessed the effect of DHEA supplementation on cycle outcome in patients with poor ovarian response. ${ }^{6}$ A favorable decrease was noted in mean day 2 serum FSH concentrations after DHEA supplementation (75.14 +/- 28.93 versus 43.07 +/- 11.77; $\mathrm{P}<0.01)$. They summed up that oral DHEA supplementation causes a reduction in the levels of basal FSH levels.

DHEA improves steroidogenesis, since it is a precursor of estradiol and testosterone. Androgens influence 
ovarian follicular growth, not only by acting as a metabolic precursor for steroid production, but also by serving as ligands for androgen receptors. In present study after administration of oral DHEA for 12 weeks, the mean S.E2 level increased from $29.66 \pm 4.9 \mathrm{mIU} / \mathrm{ml}$ to $57.67 \pm 9.6 \mathrm{mIU} / \mathrm{ml}(\mathrm{p}=0.03)$. Most importantly, the present data clearly show that oral DHEA increases the endogenous estradiol production. Similar were the results of Mamas and Mamas, they conducted study on five women with premature ovarian failure. ${ }^{7}$ The mean S. $E_{2}$ level increases from $26.4 \pm 6.65 \mathrm{mIU} / \mathrm{ml}$ to $56.6 \pm 5.27$ $\mathrm{mIU} / \mathrm{ml} \quad(\mathrm{p}=0.01)$. They concluded that oral DHEA increased the endogenous estradiol production.

$\mathrm{AMH}$, a dimeric glycoprotein and a member of the transforming growth factor superfamily, is exclusively produced by granulosa cells of early developing follicles, which are from preantral and small antral follicle stages. $\mathrm{AMH}$ may be involved in the recruitment of $\mathrm{FSH}$ sensitive follicles in the early antral stage. Thus, by improvement in S. AMH concentration DHEA progressively improves ovarian reserve in patients with diminished ovarian reserve (DOR).This means that over time DHEA increases the pool of follicles upto pre antral stage causing a steady improvement in AMH levels. A favorable increase was noted in mean S. AMH concentrations after DHEA supplementation (1.43 +/0.57 versus $0.65+/-0.30 ; \mathrm{p}<0.01)$. Gleicher et al also reported that DHEA improve oocyte/embryo yields and oocyte/embryo quality in women with DOR. ${ }^{8}$ The study was a retrospective cross-sectional and longitudinal analysis of 120 women with DOR, in whom AMH concentrations were evaluated as a reflection of ovarian reserve. AMH concentrations significantly improved after DHEA supplementation over time $(p=0.002)$. Women under age 38 years demonstrated higher AMH concentrations and improved AMH concentrations more than older females. AMH improved longitudinally by approximately $60 \% \quad(\mathrm{P}<0.0002)$. The present study showed a minimal increase in $\mathrm{S}$. Inhibin levels from $34.06 \pm 3.61$ to $37.67 \pm 4.51 \mathrm{pg} / \mathrm{ml}(\mathrm{p}=1)$.

The increase observed in antral follicle count following oral DHEA administration was not statistically significant $(6.95+/-1.61$ versus $13.05+/-1.73)(\mathrm{p}=0.56)$. The mean ovarian volume was found to be increased insignificantly from $6.3 \pm 0.95 \mathrm{ml}$ to $7.81 \pm 0.98 \mathrm{ml}$.

The number of infertile women who conceived, increased after administration of oral DHEA (49.66\% versus $13.66 \%$ ) as compared to control group and was statistically significant $(\mathrm{p}=0.04)$. In accordance to this data showing the beneficial effect of DHEA on pregnancy rate, Gleicher et al assessed the role of DHEA supplementation on pregnancy rates in women with DOR and found that cumulative clinical pregnancy rates were significantly higher in the study group (25 pregnancies; $28.4 \%$ vs. 11 pregnancies; $11.9 \%$ ( $\mathrm{p}<0.05) .{ }^{9}$ These data support a beneficial effect of DHEA supplementation among women with DOR. Gleicher et al also reported that DHEA improve oocyte/embryo yields and oocyte/embryo quality in women with DOR. ${ }^{8}$ Women reaching IVF experienced a $23.64 \%$ clinical pregnancy rate and these women showed significantly improved AMH concentrations compared with those who did not $(\mathrm{p}=0.001)$. Fusi et al also observed the unexpected spontaneous pregnancies in poor responder patients with long-term infertility, when treated with DHEA supplementation prior to IVF. ${ }^{10}$ surprisingly, spontaneous pregnancy rate significantly increased after DHEA treatment, allowing to achieve 10 spontaneous pregnancies and 9 spontaneous ongoing pregnancies among young poor responders. Pregnancy rate and ongoing pregnancy rate obtained before starting the IVF cycle were also significantly higher in older women treated with DHEA than in the control group: $21.05 \%$ and $13.15 \%$ and $4.1 \%$ and 0 , respectively $(p=0.001)$. Their results showed that DHEA supplementation improves the ovarian function in poor responders and in women over 40 years, suggesting that this molecule alone can raise fecundity and fertility treatment success in women with poor prognosis for pregnancy. There was decrease in the number of miscarriage in those treated with oral DHEA as compared to those receiving placebo but this decrease was not statistically significant. Similar were the results of Gleicher et al, they retrospectively compared, miscarriage rates in 73 DHEA supplemented pregnancies at two independent North American infertility centers, age-stratified, to miscarriages reported in a national U.S. in vitro fertilization (IVF) data base. ${ }^{11}$ After DHEA supplementation the miscarriage rate at both centers was $15.1 \%$ (15.0\% and $15.2 \%$ respectively). They concluded that DHEA supplementation improves pregnancy chances in women with DOR, by possibly reducing aneuploidy. Oral DHEA intake for 12 weeks had increased the live birth rate in study group (31.33\% versus $3.42 \%)$ as compared to control group which was statistically significant $(\mathrm{p}=0.04)$. In accordance with present study Wiser et al, evaluated the effect of DHEA supplementation on IVF data and outcomes among poorresponder patients and found that patients in the DHEA group had a significantly higher $(\mathrm{p}=0.05)$ live birth rate compared with controls $(23.1 \%$ versus $4.0 \%)$, respectively. ${ }^{12}$

\section{CONCLUSIONS}

This study concluded that DHEA supplementation can improve patient's ovarian reserve. DHEA supplementation by providing a more enhanced availability of ovarian steroidogenic prohormone, may facilitate follicular maturation and growth. The results of the study favour DHEA administration in infertile women with DOR as it has been found to improve pregnancy chances and since a large majority of spontaneous miscarriages are associated with aneuploidy, it can be assumed that DHEA supplementation may reduce miscarriage rates by reducing aneuploidy. 
Funding: No funding sources

Conflict of interest: None declared

Ethical approval: The study was approved by the Institutional Ethics Committee

\section{REFERENCES}

1. Te Velde ER, Scheffer GJ, Dorland M, Broekmans FJ, Fauser BC. Developmental and endocrine aspects of normal ovarian ageing. Molecular and cellular endocrinology. 1998a;145:67-73.

2. Hillier SG, Tetsuka M, Fraser HM. Androgen receptor function in folliculogenesis and its clinical implication in premature ovarian failure. Hum Reprod. 1997;12:107-11.

3. Weil SJ, Vendola K, Zhou J, Adesanya OO, Wang J, Bondy CA. Androgen receptor gene expression in the primate ovary: cellular localization, regulation and functional correlations. J Clin Endocrinol Metab. 1998;83:2479-85.

4. Gleicher et al. Improvement in diminished ovarian reserve after dehydroepiandrosterone supplementation. Reprod Biomed Online. 2010;21:360-5.

5. Gleicher N, Weghofer A, Barad DH. Dehydroepiandrosterone (DHEA) reduces embryo aneuploidy: direct evidence from pre implantation genetic screening (PGS). Reprod Biol Endocrinol. 2010;8:140.

6. Sonmezer M, Ozmen B, Cil AP, Ozkavukcu S, Tasci $\mathrm{T}$, Olmuş H, Atabekoglu CS. Dehydroepiandrosterone supplementation improves ovarian response and cycle outcome in poor responders. Reprod Biomed Online. 2009;19(4):50813.
7. Mamas L, Mamas E. Premature ovarian failure and dehydroepiandrosterone. Fertil Steril. 2009;91(2):644-6.

8. Gleicher N, Barad DH. Dehydroepiandrosterone (DHEA) supplementation in diminished ovarian reserve (DOR). Reprod Biol Endocrinol. 2011;17(9):67.

9. Barad DH, Brill H, Gleicher N. Update on the use of dehydroepiandrosterone supplementation among women with diminished ovarian function. J Assist Reprod Genet. 2007;24:629-34.

10. Fusi FM, Ferrario M, Bosisio C, Arnoldi M, Zanga. DHEA supplementation positively affects spontaneous pregnancies in women with diminished ovarian function. Gynecol Endocrinol. 2013;29(10):940-3.

11. Gleicher N, Ryan E, Weghofer A, Blanco S, Barad $\mathrm{DH}$. Miscarriage rates after dehydroepiandrosterone (DHEA) supplementation in women with diminished ovarian reserve: a case control study. Reprod Biol Endocrinol. 2009;7:108.

12. Wiser A, Gonen O, Ghetler Y, Shavit T, Berkovitz A, Shulman A. Addition of dehydroepiandrosterone (DHEA) for poor-responder patients before and during IVF treatment improves the pregnancy rate : a randomized prospective study. Human Reproduction. 2010;25(10):2496-2500.

Cite this article as: Dayal M, Yadav P, Chaurasia A, Singh U, Gupta V, Patel S. Role of oral dehydroepiandrosterone in diminished ovarian function. Int J Reprod Contracept Obstet Gynecol 2016;5:826-30. 